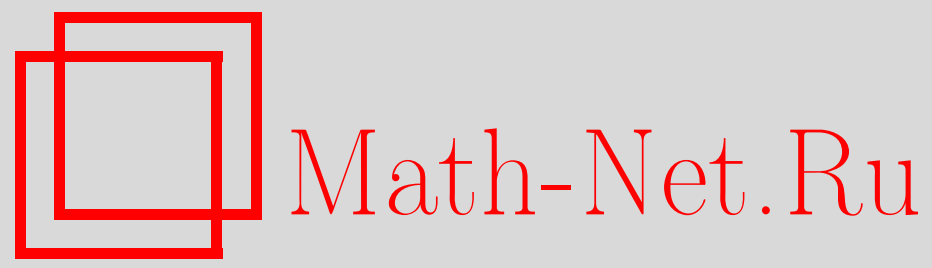

В. В. Горюнов, Простые функции на пространственных кривых, Функи. анализ и его прил., 2000, том 34, выпуск 2, 63-67

DOI: https://doi.org/10.4213/faa296

Использование Общероссийского математического портала MathNet.Ru подразумевает, что вы прочитали и согласны с пользовательским соглашением

http://www.mathnet.ru/rus/agreement

Параметры загрузки:

IP : 54.84 .234 .179

26 апреля 2023 г., 11:23:44

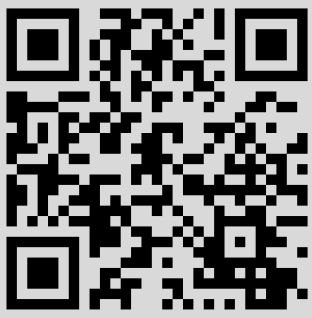




\title{
Простые функции на пространственных кривых
}

\author{
(c) 2000. В. В. Горюнов
}

Классифицируются простые особенности функций на пространственных кривых. Показано, что их бифуркационные множества обладают теми же свойствами, что и бифуркационные множества функций на гладких многообразиях и полных пересечениях $[3,4]$ : для их бифуркационных диаграмм справедлива $k(\pi, 1)$-теорема, и как бифуркационная диаграмма, так и дискриминант особенности являются свободными дивизорами в смысле Саито.

1. Пространственные кривые и функции на них. Росток приведенной пространственной кривой - детерминантное многообразие: он является множеством общих нулей всех миноров порядка $n$ ростка на $\mathbb{C}^{3}$ некоторой матрицы размера $n \times(n+1)$. В дальнейшем мы отождествляем кривую с задающей ее матрицей.

ОПРЕДЕЛЕниЕ 1.1. Два ростка кривых в начале координат, заданные матрицами $M$ и $M^{\prime}$, называются эквивалентныли, если существуют два ростка $A$ и $B$ обратимых матриц, а также росток $h$ биголоморфизма пространства $\left(\mathbb{C}^{3}, 0\right)$, такие, что $A M B=M^{\prime} \circ h$.

Простые пространственные кривые были недавно расклассифицированы в работе [8].

Мы будем говорить, что коранг ростка пространственной кривой равен $c$, если ранг задающей его матрицы в начале координат равен $n-c$. Такая кривая может быть задана ростком матрицы размера $c \times(c+1)$, значение которого в начале координат есть нулевая матрица.

ОПРедЕЛЕниЕ 1.2. Росток на $\left(\mathbb{C}^{3}, 0\right)$ пары $(M, f)$, где $M$ - матрица размера $n \times(n+1)$, а $f$ - функция, называется функцией на пространственной кривой.

ОПреДЕЛЕниЕ 1.3. Ростки $(M, f)$ и $\left(M^{\prime}, f^{\prime}\right)$ функций в начале координат называются $\mathscr{R}_{c}$-эквивалентныли, если существуют ростки $A$ и $B$ обратимых матриц, росток $h$ биголоморфизма пространства $\left(\mathbb{C}^{3}, 0\right)$, а также функция $g$ из идеала, порожденного максимальными минорами матрицы $M$, такие, что

$$
(A M B, f+g)=\left(M^{\prime} \circ h, f^{\prime} \circ h\right)
$$

Понятие $\mathscr{R}_{c}$-эквивалентности удовлетворяет всем условиям хорошей геометрической эквивалентности Деймона [6]. Поэтому для нее справедливы все стандартные теоремы типа теорем версальности и конечной определенности.

\section{2. Простые особенности.}

Теорема 2.1. Простая функция на пространственной кривой - это функиия или на плоской кривой, или на кривой коранга 2 . В соответствии с этим полный список простых функций состоит из двух частей: 


\begin{tabular}{|c|c|c|c|c|}
\hline $\begin{array}{c}\text { обозна- } \\
\text { чение }\end{array}$ & $\begin{array}{c}\text { уравнения } \\
\text { кривой }\end{array}$ & функция & ограничения & индекс \\
\hline$A_{k}$ & $y$ & $x^{k+1}$ & $k \geqslant 0$ & $(k+1)^{k-1}$ \\
$C_{p, q}$ & $x y$ & $x^{p}+y^{q}$ & $p \geqslant q \geqslant 1$ & $\frac{(p+q-1) ! p^{p} q^{q}}{(p-1) !(q-1) !}$ \\
$B_{k}$ & $x^{2}+y^{k}$ & $y$ & $k \geqslant 3$ & 1 \\
$F_{k}$ & $x^{2}+y^{3}$ & $\left\{\begin{array}{cc}y^{r} \\
x y^{r}\end{array}\right.$ & $\begin{array}{c}k=2 r+1 \geqslant 5 \\
k=2 r+4 \geqslant 4\end{array}$ & $\frac{(k-2)(k-1)^{k} k}{24}$ \\
\hline$C_{p, q, r}$ & $\left|\begin{array}{ccc}x & y & 0 \\
0 & y & z\end{array}\right|$ & $x^{p}+y^{q}+z^{r}$ & $p \geqslant q \geqslant r \geqslant 1$ & $\frac{(p+q+r+1) ! p^{p} q^{q} r^{r}}{(p-1) !(q-1) !(r-1) !}$ \\
$\dot{F}_{k}$ & $\left|\begin{array}{ccc}x & y & 0 \\
y^{2} & x & z\end{array}\right|$ & $z+\left\{\begin{array}{c}y^{r} \\
x y^{r}\end{array}\right.$ & $k=2 r+3 \geqslant 5$ & $\frac{(k-3)^{k}(k-2)(k-1) k}{24}$ \\
$\check{E}_{6}$ & $\left|\begin{array}{lll}x & y & z \\
z^{2} & x & y\end{array}\right|$ & $z$ &
\end{tabular}

Первая половина списка была получена в [7]. Смысл последнего столбца будет разъяснен в разд. 4.

Все примыкания табличных функций на плоских кривых являются композициями примыканий внутри серий, получаемых уменьшением индексов (удобно считать, что $B_{2}=C_{1,1}$ и $\left.F_{3}=B_{3}\right)$, а также примыканий

$$
F_{p+q+1} \rightarrow C_{p, q} \rightarrow A_{p+q-1}
$$

Аналогично, уменьшение индексов в сериях дает очевидные примыкания особенностей во второй половине таблицы. Отметим также некоторые другие примыкания, дающие представление о взаимодействии серий:

$$
C_{p, q, r} \rightarrow C_{p, q}, \quad \dot{F}_{k} \rightarrow F_{k-2}, \quad \dot{F}_{p+q+3} \rightarrow C_{p, q, 1}, \quad \check{E}_{6} \rightarrow \dot{F}_{5} .
$$

Любая непростая функция - это или функция на кривой коранга больше двух, или особенность, примыкающая к одной из двух непростых функций на плоских кривых:

$$
\begin{array}{lll}
X_{9}^{*}: & x+\alpha y^{2} & \text { на } x^{2}+y^{4}=0, \\
J_{10}^{*}: & x+\alpha y & \text { на } x^{3}+y^{3}=0 .
\end{array}
$$

Здесь общее комплексное число $\alpha$ является модулем. Обе особенности примыкают к $B_{4}$ и $F_{4}$. Кроме того, $X_{9}^{*}$ примыкает к $C_{3,1}$.

3. Бифуркационные многообразия как свободные дивизоры. Пусть $\left(x_{1}, x_{2}, x_{3}\right)$ - координаты, а $\mathscr{O}_{3}$ - пространство ростков голоморфных функций на $\left(\mathbb{C}^{3}, 0\right)$. Отождествим пространство ростков пар, состоящих из $n \times$ $(n+1)$-матрицы и функции, с модулем $\mathscr{O}_{3}^{n(n+1)+1}$. Касательное пространство 
$T(M, f)$ к классу (расширенной) $\mathscr{R}_{c}$-эквивалентности ростка $(M, f)$ в этом модуле - это $\mathscr{O}_{3}$-подмодуль, порожденный элементами

$$
\begin{array}{ll}
\left(E_{i j}^{n} M, 0\right), & i, j=1, \ldots, n, \\
\left(M E_{k l}^{n+1}, 0\right), & k, l=1, \ldots, n+1, \\
\left(0, \varphi_{r}\right), & r=1, \ldots, n+1, \\
\left(\partial M / \partial x_{s}, \partial f / \partial x_{s}\right), & s=1,2,3 .
\end{array}
$$

Здесь $E_{i j}^{n}$ есть $n \times n$-матрица, у которой на пересечении $i$-й строки и $j$-го столбца стоит 1 , а на остальных местах — нули, а функции $\varphi_{r}$ - максимальные миноры матрицы $M$.

Положим $\tau(M, f)=\operatorname{dim}_{\mathbb{C}} \mathscr{O}_{3}^{n(n+1)+1} / T(M, f)$ и назовем это число числом Тюриной особенности. Число Тюриной — это размерность базы $\mathscr{R}_{c}$-миниверсальной деформации функции $(M, f)$. Такая деформация может быть представлена в форме $(M, f)+\lambda_{1} e_{1}+\cdots+\lambda_{\tau} e_{\tau}$, где $\lambda_{i}-$ параметры, а $e_{i}-$ элементы модуля $\mathscr{O}_{3}^{n(n+1)+1}$, проектирующиеся в базис линейного пространства $\mathscr{O}_{3}^{n(n+1)+1} / T(M, f)$.

$\mathrm{У}$ особенностей $C_{p, q}$ и $C_{p, q, r}$ числа Тюриной соответственно равны $p+q$ и $p+q+r+1$. У остальных простых особенностей $\tau$ - нижний индекс в обозначении. Кроме того, $\tau\left(X_{9}^{*}\right)=\tau\left(J_{10}^{*}\right)=6$.

Обозначим через $\mu(M, f)$ число морсовских критических точек общего малого возмущения функции $f$ на общем сглаживании кривой $M$. Назовем $\mu(M, f)$ числом Милнора особенности.

ГипотеЗА 3.1. $\tau(M, f)=\mu(M, f)$.

Эта гипотеза справедлива для функций на полных пересечениях [7], для $\mathscr{R}_{c}$-простых особенностей, а также в некоторых других частных случаях.

ОПредЕЛЕниЕ 3.2. Рассмотрим базу $\mathbb{C}^{\tau} \mathscr{R}_{c}$-миниверсальной деформации особенности $(M, f)$. Дискриминантом $\Delta(M, f) \subset \mathbb{C}^{\tau}$ особенности $(M, f)$ называется множество значений параметров деформации, отвечающих функциям на кривых с нулевым критическим значением. Бифуркачионной диаграммой $\oint$ фкиий $\Sigma(M, f) \subset \mathbb{C}^{\tau}$ особенности $(M, f)$ называется множество значений параметров, отвечающих функциям на кривых с менее, чем $\mu(M, f)$, различными критическими значениями.

Заметим, что особая точка кривой должна рассматриваться как критическая для функции на этой кривой.

ОПредЕЛЕниЕ 3.3. Гиперповерхность $H$ в $\mathbb{C}^{N}$ называется свободнылм дивизором, если алгебра $\Theta_{H}$ векторных полей на $\mathbb{C}^{N}$, касающихся $H$ (т. е. сохраняющих идеал этой гиперповерхности), порождена (как модуль над функциями на $\left.\mathbb{C}^{N}\right) N$ элементами.

ТЕорема 3.4. Предположим, что $\tau(M, f)=\mu(M, f)$. Тогда как дискриминант $\Delta(M, f) \subset \mathbb{C}^{\tau}$, так и бифуркачионная диаграмма функиий $\Sigma(M, f) \subset \mathbb{C}^{\tau}$ являются свободньми дивизорами.

Системы образующих модулей $\Theta_{\Delta}$ и $\Theta_{\Sigma}$ можно построить следующим способом (ср. $[10,5,4])$. Пусть $(\mathscr{M}, F)=(\mathscr{M}(x, \lambda), F(x, \lambda))$ есть $\mathscr{R}_{c}$-миниверсальная 
деформация особенности $(M, f)$ с параметрами $\lambda=\left(\lambda_{1}, \ldots, \lambda_{\tau}\right) \in \mathbb{C}^{\tau}$. В силу версальности деформации для всякого $i=1, \ldots, \tau$ существуют разложения

$$
\begin{gathered}
F \frac{\partial}{\partial \lambda_{i}}(\mathscr{M}, F)=\left(\mathscr{A}_{i} \mathscr{M} \mathscr{B}_{i}, \mathscr{G}_{i}\right)+\sum_{s=1}^{3} h_{i s} \frac{\partial}{\partial x_{s}}(\mathscr{M}, F)+\sum_{j=1}^{\tau} v_{i j} \frac{\partial}{\partial \lambda_{j}}(\mathscr{M}, F), \\
F^{i-1} \frac{\partial}{\partial \lambda_{i}}\left(\mathscr{M}, F^{\prime}\right)=\left(\mathscr{A}_{i}^{\prime} \mathscr{M} \mathscr{B}_{i}^{\prime}, \mathscr{G}_{i}^{\prime}\right)+\sum_{s=1}^{3} h_{i s}^{\prime} \frac{\partial}{\partial x_{s}}(\mathscr{M}, F)+\sum_{j=1}^{\tau} w_{i j} \frac{\partial}{\partial \lambda_{j}}(\mathscr{M}, F),
\end{gathered}
$$

где $\mathscr{A}_{i}(x, \lambda)$ и $\mathscr{B}_{i}(x, \lambda)$ - ростки матриц, $h_{i s}(x, \lambda)$ и $v_{i j}(\lambda)-$ ростки функций, $\mathscr{G}_{i}(x, \lambda)$ - элемент идеала, порожденного максимальными минорами матрицы $\mathscr{M}$ в кольце функций от $x$ и $\lambda$, и аналогичные обозначения использованы во второй формуле. Наличие функционального множителя или знака дифференцирования перед парой (матрица, функция) означает, что оба члена должны быть умножены на данный множитель или же продифференцированы.

Векторные поля

$$
\nu_{i}=\sum_{j=1}^{\tau} v_{i j}(\lambda) \partial_{\lambda_{j}} \quad \text { и } \quad \omega_{i}=\sum_{j=1}^{\tau} w_{i j}(\lambda) \partial_{\lambda_{j}}
$$

образуют базисы соответственно модулей $\Theta_{\Delta}$ и $\Theta_{\Sigma}$.

4. Отображение Ляшко-Лойенги. Рассмотрим пространство $\mathbb{C}^{\mu}$ всех полиномов степени $\mu$ от одной переменной со старшим коэффициентом единица. Обозначим через $\Xi \subset \mathbb{C}^{\mu}$ множество полиномов с кратными корнями.

Фиксировав особенность $(M, f)$ с числом Тюриной $\tau$ и числом Милнора $\mu$, рассмотрим отображение $\mathbb{C}^{\tau} \backslash \Sigma(M, f) \rightarrow \mathbb{C}^{\mu} \backslash \Xi$ дополнения к бифуркационной диаграмме функций, которое ставит в соответствие морсовской функции на гладкой кривой неупорядоченное множество ее критических значений, т. е. полином, корнями которого эти критические значения и являются. Наше отображение, как нетрудно проверить, продолжается до отображения объемлющих комплексных линейных пространств. Назовем полученное продолжение отображением Ляшко-Лойенги.

ТЕорема 4.1. Отображение Ляшко-Лойенги $\mathscr{R}_{c}$-простой функции на пространственной кривой является конечнократным накрытием. Как отображение из $\mathbb{C}^{\tau} \backslash \Sigma(M, f)$ в $\mathbb{C}^{\mu} \backslash \Xi$ оно неразветвленное.

СледСтвиЕ 4.2. Дополнение $\kappa$ бифуркачионной диаграмме функиий $\mathscr{R}_{c}$-простой особенности $(M, f)$ в базе ее $\mathscr{R}_{c}$-миниверсальной деформачии является пространством типа $k(\pi, 1)$, где $\pi$ - подгруппа конечного индекса в группе кос из $\mu(M, f)$ нитей.

Значения индекса приведены в последнем столбце классификационной таблицы. Теорема 4.1 и ее следствие аналогичны классической теореме о простых функциях на гладких многообразиях $[1,9,2,3]$ и обобщают соответствующие утверждения о простых функциях на плоских кривых $[7,4]$.

\section{ЛИТЕРАТУРА}

1. Арнольд В. И. УМН, 29, вып. 3, 243-244 (1974). 2. Арнольд В. И., Варченко А. Н., Гусейн-Заде С. М. Особенности дифференцируемых отображений. II. Наука, М., 
1984. 3. Арнольд В. И., Васильев В. А., Горюнов В. В., Ляшко О. В. Особенности I. Локальная и глобальная теория. Динамические системы VI. Итоги науки и техники. Соврем. пробл. матем. Фундам. напр., т. 6, ВИНИТИ, М., 1988. 4. Арнольд В. И., Васильев В. А., Горюнов В. В., Ляшко О. В. Особенности II. Классификация и приложения. Динамические системы VIII. Итоги науки и техники. Соврем. пробл. матем. Фундам. напр., т. 39, ВИНИТИ, М., 1989. 5. Bruce J. W. Bull. London Math. Soc., 17, No. 5, 257-262 (1985). 6. Damon J. N. Mem. Amer. Math. Soc., 50, No. 306, 1-88 (1984). 7. Горюнов B. В. Функц. анализ и его прил., 15, вып. 2, 1-8 (1981). 8. Frühbis-Krueger A. Preprint, Universität Kaiserslautern, 1998. 9. Looijenga E. J. N. Invent. Math., 23, 105-116 (1974). 10. Закалюкин B. M. Функц. анализ и его прил., 10, вып. 2, 69-70 (1976).

Department of Mathematical Sciences

Division of Pure Mathematics

Поступило в редакцию

The University of Liverpool

8 сентября 1998 г.

e-mail: goryunov@liv.ac.uk

УДК 517.98

\title{
Туннельное расщепление спектра операторов Лапласа-Бельтрами на двумерных поверхностях с квадратично интегрируемым геодезическим потоком
}

\author{
(C) 2000. С. Ю. Доврохотов, А. И. ШАФАРЕВИЧ
}

Пусть $M$ - тор или сфера с квадратично интегрируемым по Лиувиллю геодезическим потоком, т. е. в кокасательном расслоении $T^{*} M$ существует независимый с гамильтонианом $H=|p|^{2}$ квадратичный по импульсам $p$ интеграл $F$ (см. [1-3]). Метрика на $M$ приводится к лиувиллеву виду $d s^{2}=$ $\left(u_{1}\left(x_{1}\right)-u_{2}\left(x_{2}\right)\right)\left(d x_{1}^{2}+d x_{2}^{2}\right), u_{1}-u_{2}>0$, причем координаты $\left(x_{1}, x_{2}\right)$ определены на торе $\widetilde{M}$, конечнолистно накрывающем исходную поверхность $M$. Пусть $\widetilde{F}, \widetilde{H}$ — поднятия интегралов $F, H$ на $T^{*} \widetilde{M}$. Множество уровня $\{\widetilde{H}=E, \widetilde{F}=\mathscr{E}\}$, где $\min u_{1}<\mathscr{E} / E<\max u_{1}$, состоит из двух торов $\widetilde{\Lambda}^{ \pm}$, накрывающих лиувиллевы торы геодезического потока. Инволюция $p \rightarrow-p$ переводит в себя лиувиллево слоение, причем отдельные лиувиллевы торы, вообще говоря, переставляются между собой.

Пусть $\Delta$ - оператор Лапласа-Бельтрами на $M$; каждый неособый лиувиллев тор $\Lambda$ геодезического потока, удовлетворяющий условию квантования [5], определяет в квазиклассическом пределе $h \rightarrow 0$ квазимоду $\psi \in L^{2}(M), E \in \mathbb{R}$ оператора $-h^{2} \Delta$. При этом асимптотическая собственная функция $\psi$ имеет вид $\psi=K_{\Lambda}^{a}(1)$, где $K_{\Lambda}^{a}$ - канонический оператор Маслова [5] на лагранжевом торе $\Lambda$ с произвольной отмеченной точкой $a$; асимптотическое собственное число $E$ вычисляется из условий квантования (явные формулы для случая $M=T^{2}$ см., например, в [6]). Рассмотрим пару различных торов $\Lambda^{ \pm}$, переходящих друг в друга под действием инволюции $p \rightarrow-p$. Они одновременно удовлетворяют (или не удовлетворяют) условиям квантования, причем числа $E$, определяемые этими торами, совпадают. Переменные в спектральной задаче для оператора Лапласа разделяются; используя результаты [4], нетрудно доказать существо- 